\title{
Targeted disruption of the mouse testis-enriched gene Znf230 does not affect spermatogenesis or fertility
}

\author{
Yunqiang Liu, Dachang Tao, Yongjie Lu, Yuan Yang, Yongxin Ma and Sizhong Zhang \\ Department of Medical Genetics and Division of Human Morbid Genomics, State Key Laboratory of \\ Biotherapy, West China Hospital, West China Medical School, Sichuan Universtiy, Chengdu, Sichuan \\ Province, China.
}

\begin{abstract}
The mouse testis-enriched Znf230 gene, which encodes a type of RING finger protein, is present primarily in the nuclei of spermatogonia, the acrosome and the tail of spermatozoa. To investigate the role of Znf230 in spermatogenesis, we generated Znf230-deficient mice by disrupting Znf230 exon-5 and exon-6 using homologous recombination. The homozygous Znf230-knockout (KO) mice did not exhibit Znf230 mRNA expression and Znf230 protein production. Znf230 KO mice exhibited no obvious impairment in body growth or fertility. Male Znf230 KO mice had integral reproductive systems and mature sperm that were regular in number and shape. The developmental stages of male germ cells of Znf230 KO mice were also normal. We further examined variations in the transcriptomes of testicular tissue between Znf230 KO and wild-type mice through microarray analysis. The results showed that the mRNA level of one unclassified transcript 4921513I08Rik was increased and that the mRNA levels of three other transcripts, i.e., 4930448A20Rik, 4931431B13Rik and potassium channel tetramerisation domain containing 14(Kctd14), were reduced more than two-fold in Znf230 KO mice compared with wild-type mice. Using our current examination techniques, these findings suggested that Znf230 deficiency in mice may not affect growth, fertility or spermatogenesis.
\end{abstract}

Keywords: Znf230, knockout mice, spermatogenesis, Kctd14.

Received: October 9, 2013; Accepted: June 24, 2014.

\section{Introduction}

Mammalian spermatozoa development is a complex process that involves the renewal and differentiation of spermatogonia, the meiosis of spermatocytes, and drastic morphological changes accompanying the development from round spermatids to mature spermatozoa (Russell et al., 1990). Many environmental, behavioral and genetic factors affect male fertility. An estimated $50 \%$ of human infertility has been attributed to genetic abnormalities (Hwang et al., 2011). Two previous microarray studies (Schultz et al., 2003; Schlecht et al., 2004) showed that haploid germ cells express a large number of germ cellspecific genes (approximately 4\% of mammalian genes). Therefore, it is necessary to identify these unique genes and characterize the precisely programmed cell- and stagespecific gene expression that occurs during the regulation of the developmental spermatogenesis process.

Send correspondence to Yunqiang Liu. Department of Medical Genetics and Division of Human Morbid Genomics, State Key Laboratory of Biotherapy, West China Hospital, West China Medical School, Sichuan University, No. 1, Ke Yuan 4 Lu, Gao Peng Da Dao, Chengdu, 610041, Sichuan, China. E-mail: yq_liu@scu.edu.cn.
The human gene ZNF230 (also named RNF141), which encodes a type of RING (Really Interesting New Gene) finger protein, was first identified in our laboratory to be restrictively expressed in the testicular tissue of fertile men (Zhang et al., 2001). RING finger proteins, a subfamily of zinc finger proteins (ZFP), often contain the cysteine-rich $\mathrm{CX}_{2} \mathrm{CX}_{(9-39)} \mathrm{CX}_{(1-3)} \mathrm{HX}_{(2-3)} \mathrm{CX}_{2} \mathrm{CX}_{(4-48)} \mathrm{CX}_{2} \mathrm{C}$ domain and are involved in a variety of biological processes, including transcriptional regulation, signal transduction, cell apoptosis and protein ubiquitination (Borden, 2000; Joazeiro and Weissman, 2000). The mouse homolog of Znf230 was also identified in our laboratory (Qiu et al., 2003). The expression of the mouse Znf230 gene is developmentally regulated, and the $\mathrm{Znf} 230$ protein functions as an activator module in transcription. Additionally, the mouse Znf230 protein is primarily expressed in the nuclei of spermatogonia but has subsequent expression in the acrosome system and the tails of developing spermatids and spermatozoa (Song et al., 2008). Hence, we wondered whether Znf230 may play a role in mammalian spermatogenesis.

Animal models have defined key signaling pathways that are involved in reproductive physiology ( $\mathrm{Li}$ et al., 2001). To date, over 400 genes that are essential for male 
fertility have been identified using transgenic, chemically induced, point mutants and $\mathrm{KO} / \mathrm{knock}$-in/gene-trap mouse models (Yatsenko et al., 2010; Jamsai and O’Bryan, 2011).

To investigate the role of Znf230 in mouse spermatogenesis, we used a targeted gene KO strategy to generate Znf230-deficient mice. We had previously constructed a gene-targeting vector based on a modified pPNT vector and generated mutant mice with exon-2 of the Znf230 gene disrupted (Liu et al., 2013). However, a partial sequence from the pPNT vector acted as an alternative exon-2, thus allowing a new Znf230 transcript to be produced in the mutant mice and a new protein product, possessing a C-terminal amino acid sequence with a RING finger motif similar to that of the wild-type $\mathrm{Znf} 230$ protein, to be generated. Thus, Znf230 function in the mutant mice was not entirely inactivated. In the current study, we changed the targeting strategy such that the region of exon-5 and exon-6, which encodes the essential RING finger domain of the Znf230 protein, was directly disrupted. This strategy successfully generated Znf230-null mice for use in this study.

\section{Materials and Methods}

\section{Construction of Znf230 KO targeting vector and generation of Znf230 KO mice}

Using a highly efficient recombineering-based method that has been previously described (Liu et al., 2003; Chan et al., 2007), the Znf230 KO targeting vector was constructed from a genomic DNA fragment derived from the $\mathrm{C} 57 \mathrm{BL} / 6 \mathrm{~J}$ bacterial artificial chromosome clone bMQ-291L21. The targeting construct, which had a 4864 bp left arm containing introns 2 and 3 of the Znf230 gene and a 2902 bp right arm containing the partial untranslated region of exon-6, was inserted into the ABRLFn-pBR32 vector (Figure 1).

Thirty micrograms of the targeting vector was linearized by Not I and transfected into CJ7 (derived from $129 \mathrm{SV} / \mathrm{J}$ mice) embryonic stem cells (ESCs) by electroporation. Ninety-six ESC clones were selected with $300 \mu \mathrm{g} / \mathrm{mL}$ G418 (Geneticin, Sigma-Aldrich Co., St. Louis, MO, USA). Among these neomycin-resistant cells, 12 ESCs that had undergone homologous recombination were identified by long polymerase chain reaction (L-PCR) analysis with two pairs of primers P1F: 5'-acctctggcctttacaaactcatg-3', 5 '-ggcetaccegcttccattgctc-3' and P1R: 5' -ccgtgecttcettgaccetgg-3', P2R: 5'-caagcagccttattacccagttg-3'.

Two correctly targeted ESC clones were microinjected into $\mathrm{C} 57 \mathrm{BL} / 6 \mathrm{~J}$ blastocysts to generate chimeras that were then crossed into a $\mathrm{C} 57 \mathrm{BL} / 6 \mathrm{~J}$ genetic background. The offspring were screened by L-PCR analysis of their genomic DNA using $\mathrm{P} 1 \mathrm{~F} / \mathrm{R}$ and $\mathrm{P} 2 \mathrm{~F} / \mathrm{R}$ primers.

The Znf230 KO mice were generated at the Shanghai Research Center for Model Organisms, Shanghai, China.

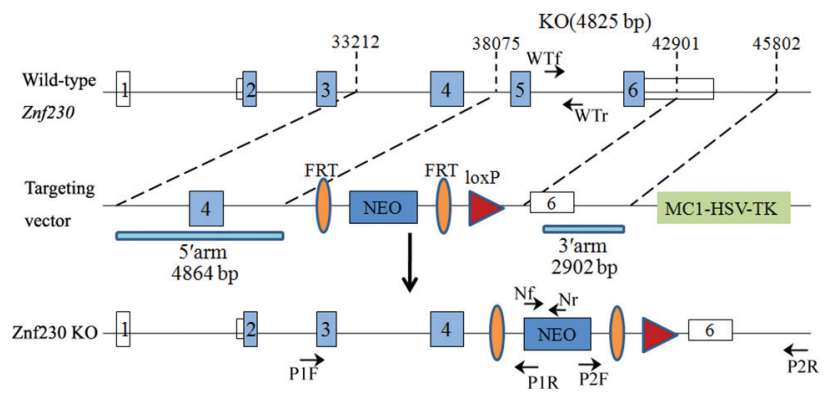

Figure 1 - The targeted knockout strategy for the $Z n f 230$ gene: wild-type allele, targeting construct and targeted allele with the location of primers. Primer pairs P1F/P1R and P2F/P2R, respectively, which flanked the targeted region, were used to select positive ESC clones, and were used to monitor the inheritance of the mutant allele. Primers WTf and WTr were used to amplify the wild-type Znf 230 allele. Primers $\mathrm{Nf}$ and $\mathrm{Nr}$ were used to amplify the knockout allele.

Znf230 KO mice were crossed into a C57BL/6J background for at least eight generations before use. Germline transmission of the targeted allele was monitored by PCR with primers including Znf230 wild type (WT)-specific primers: WTf: 5'-tgeccettgeccccataat-3', WTr: 5'-gccacccaagaaaaagtcaaaata-3' and Znf230 KO-specific primers: Nf: 5'-ggcgcgagcccetgatgctc-3', $\mathrm{Nr}$ : 5'-ttgggtggagaggctattcggctatgac-3', respectively.

The locations of the aforementioned primers are shown in Figure 1.

All animals used in this study were handled in compliance with the National Cancer Center Research Institute's guidelines for the use of animals (USA). All animal experimental protocols were approved by the animal ethics committee of West China Hospital, Sichuan University.

\section{Reverse transcription (RT)-PCR analysis}

Total RNA was extracted from the testes of at least five 15-week-old mice per genotype using an RNApure kit (Bioteke, Beijing, China). One microgram of total RNA was reverse transcribed using a RevertAid First Strand cDNA Synthesis Kit (Fermentas, Pittsburgh, PA, USA) with oligo(dT) primers. The sequence of interest in the Znf230 gene was amplified using two gene-specific primers: E4f: 5'-cccatcctcggtcacatctt-3', located within the sequence of Exon-4, and E6r: 5'-cccccttctcctctacgacaac-3', the reverse complement primer of a sequence located within Exon-6. A 982 bp fragment corresponding to the mouse Gapdh gene was co-amplified as an internal control using the following primers: 5'-tgaaggtcggtgtgaacggatttggc-3' (Forward) and 5'-catgtaggccatgaggtccaccac-3' (Reverse). Three independent RT-PCR analyses were performed to validate the results.

\section{Western blot analysis}

The testicular tissues of at least five 15-week-old mice per genotype were removed and homogenized in a 
RIPA lysis buffer containing a $1 \mu \mathrm{L} / \mathrm{mL}$ protease inhibitor cocktail (Sigma) to obtain cell lysates. After centrifugation, the supernatants were examined for protein concentration, subjected to $12 \%$ SDS-PAGE and transferred to Immobilon-P transfer membranes (Millipore, Bedford, MA, USA). The membranes were incubated with antiZnf230 primary antibody (Catalog number: ab4542, Abcam, Cambridge, MA, USA) at $4{ }^{\circ} \mathrm{C}$ overnight, washed with 1 X PBS containing $0.1 \%$ Triton X-100, and incubated with horseradish peroxidase-linked secondary antibodies (1:10,000, Boster, Wuhan, China) at room temperature for $1 \mathrm{~h}$. The respective bands were visualized using an immunostaining kit (Millipore). The anti-Znf230 primary antibody recognizes amino acids 218-230 in the C-terminus of the Znf230 protein. The quality of the antibody was validated by the manufacturer and our previous report (Song et $a l ., 2008)$. The western blot analysis was repeated again.

\section{Morphological examination}

Testes from three mice per post-natal day $(4,8$, and 15 weeks of age) were necropsied for histopathological examination. The tissues were routinely processed, embedded in paraffin and stained with hematoxylin and eosin (H\&E). Three slides per testis were visualized using a Zeiss Axio Imager Z2 microscope (Carl Zeiss, Jena, Germany).

\section{Analysis of sperm characteristics}

Cauda epididymides from five 15 -week-old mice per genotype were dissected and immediately mined in $1 \mathrm{X}$ PBS solution ( $\mathrm{pH}$ 7.4). Sperm were squeezed out with fine forceps and allowed to disperse in PBS at room temperature for $15 \mathrm{~min}$, followed by repeated pipetting. Thereafter, the sperm remaining as a mono-dispersed suspension were counted using a hemocytometer. Sperm counting was performed four times for each sample.

In addition, the sperm were washed three times in $1 \mathrm{X}$ PBS by centrifugation at $500 \mathrm{~g}$ for $5 \mathrm{~min}$ and then air-dried onto microscope slides. The slides were then stained with $\mathrm{H} \& \mathrm{E}$ and visualized using a Zeiss Axio Imager Z2 microscope.

\section{Serum sex hormone assay}

Blood was taken from five 15 -week-old male mice per genotype that were generated from different breeding pairs and housed singly in separate cages. The serum testosterone, follicle stimulating hormone (FSH), and luteinizing hormone (LH) levels were measured using an ELISA kit (Uscn Life Science Inc., Wuhan, China), according to the manufacturer's instructions.

\section{Microarray analysis}

One hundred micrograms of total RNA from testis tissues from three Znf230 KO and three C57BL/6J wildtype mice was used to generate biotin-labeled cRNA by using a Message Amp Premier RNA Amplification Kit
(Ambion, Austin, TX, USA). Following fragmentation, the labeled cRNA of each sample was hybridized to Affymetrix GeneChip ${ }^{\circledR}$ Mouse Genome 430 2.0 Arrays (Santa Clara, CA, USA) and stained according to the manufacturer's instructions.

Processed arrays were scanned using a GeneChip ${ }^{\circledR}$ Scanner 3000 (Affymetrix). The Affymetrix GeneChip ${ }^{\circledR}$ Command Console ${ }^{\circledR}$ Software was used to perform quality assessments and produce data reports. Differences in the expression patterns between groups were analyzed using the $\mathrm{R}$ program for significance analysis of microarrays (Irizarry et al., 2003).

Microarray analysis was performed at the CapitalBio Corporation, Beijing, China.

\section{Quantitative real time PCR (qRT-PCR)}

qRT-PCR analyses were performed with cDNA using a SYBR ${ }^{\circledR}$ Premix Ex Taq ${ }^{\mathrm{TM}}$ II kit (Takara, Dalan, China) and $10 \mathrm{mM}$ of the corresponding set of sense and antisense primers of the Kctd14 gene: 5'-atgggcaccctgatgaagc -3' (Forward) and 5'-gcccagtgcgcaggtagtc -3' (Reverse). The reactions were run on a Bio-Rad MyiQ Cycler (Hercules, CA, USA) using the following parameters: 40 cycles of $95^{\circ} \mathrm{C}$ for $1 \mathrm{~min}, 95^{\circ} \mathrm{C}$ for $10 \mathrm{~s}$ and $60^{\circ} \mathrm{C}$ for $30 \mathrm{~s}$. Three independent assays were performed in duplicate on each sample. The CT values from each run were averaged per sample. The $\Delta \Delta \mathrm{CT}$ method was applied for data analysis. To do so, the CT data for the Kctd14 mRNA samples were normalized with that of $\beta$-actin (Primers: 5' -aacagtccgectagaagca-3' (Forward) and 5'cgttgacatccgtaaagacc-3' (Reverse)). All qRT-PCR data are shown as the mean \pm standard deviation (S.D.). The testis sample from Znf230 KO mice was set at 1 as arbitrary unit or $100 \%$.

\section{Statistical analysis}

Student's $t$-test was used to compare data between wild-type and mutant mice. A p $<0.05$ indicated significance. All data analyses were performed using SPSS v17.0.

\section{Results}

\section{Generation of Znf230 KO mice}

A gene-targeting vector was constructed to delete the genomic segment of Znf230 that includes exon-5 and exon-6, which encode the RING finger motif of Znf230 (Figure 1). The targeting vector was introduced into ESCs. ESCs with successful homologous recombination were confirmed by two L-PCR analyses with primers flanking the targeting region (Figure 2A). The chimeric mice derived from the targeted ESCs transmitted the disrupted Znf230 allele to their offspring (Figure 2B-D).

RT-PCR analysis was performed to evaluate $Z n f 230$ gene expression, which should be disrupted in $\mathrm{Znf} 230 \mathrm{KO}$ mice. As shown in Figure 2E, the targeting region, i.e., 

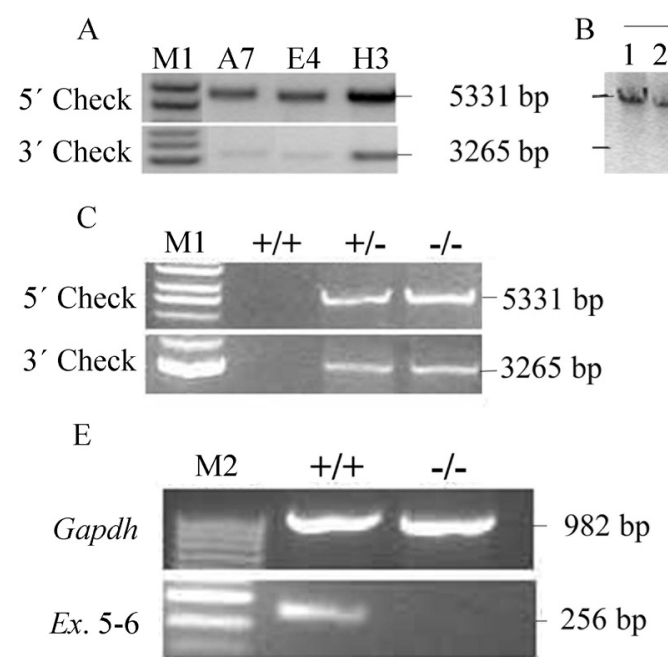

B 5' Check 3 Check

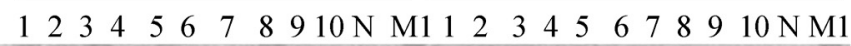

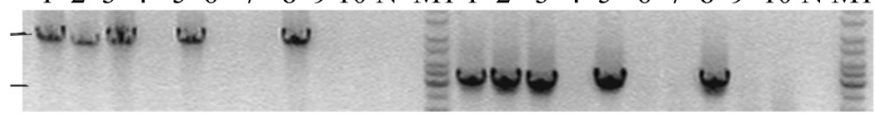

D

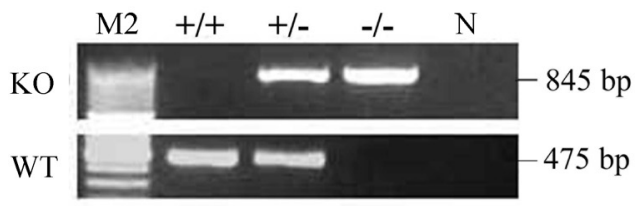

F

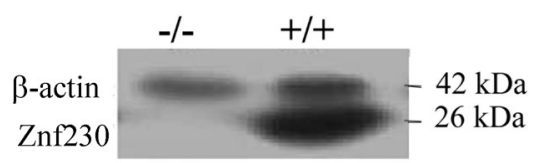

Figure 2 - The generation and identification of Znf230 KO mice. (A) L-PCR analysis using primer pairs P1F/P1R and P2F/P2R to amplify the targeted Znf230 alleles from genomic DNA extracted from ESCs. ESCs from No. A7, E4 and H3 were the positive clones undergoing targeted homologous recombination. (B) L-PCR analysis of the targeted $Z n f 230$ alleles amplified from genomic DNA derived from the offspring of chimeric mice backcrossed to C57BL/6J. No. 1-10: the offspring members and N: negative control. (C) L-PCR analysis to monitor the inheritance of the targeted Znf 230 allele in the progeny of heterozygous Znf230 KO mice. (D) PCR analysis to monitor the inheritance of the targeted Znf230 allele in the progeny of heterozygous Znf230 KO mice using primer pairs WTf/WTr and Nf/Nr. (E) RT-PCR analysis of the Znf230 gene in testes from Znf230 KO and C57BL/6J mice. M1, M2: DNA ladders of $1 \mathrm{~kb}$ and $100 \mathrm{bp}$, respectively. Gapdh was used as an internal control. (F) Western blot analysis of the Znf230 protein in the testes from $\mathrm{Znf} 230 \mathrm{KO}$ and $\mathrm{C} 57 \mathrm{BL} / 6 \mathrm{~J}$ mice. $\beta$-actin was used as an internal control.

exon-5 and exon-6, of the Znf230 gene was not amplified from total RNA of the testes in Znf230 KO mice using the specific primers, in contrast to wild-type mice. Furthermore, western blot analysis with anti-Znf230 antibody showed that the 26-kDa protein representing Znf230 was present in the testes of wild-type mice, as expected, but it was absent in the testes of Znf230 KO mice (Figure 2F). Thus, the Znf230 gene was indeed disrupted in Znf230 KO mice.

\section{Znf230 KO mice appeared to be normal in growth and fertility}

Znf230 KO mice exhibited no obvious impairment in body growth and development because no significant differences were observed in the weights of body or organs, including brain, lung, heart, liver, spleen and kidney, or life spans between Znf230 KO and wild-type mice (Table 1). Because Znf230 was identified as a testis-enriched gene that likely plays a role in male fertility, we focused our investigation on phenotypes related to male fertility. However, the fertility of Znf $230 \mathrm{KO}$ mice appeared to be normal and the offspring of Znf230 KO intercrosses were born at the expected Mendelian ratios. Compared with wild-type mice, Znf230 KO mice displayed no detectable differences in the male reproductive system, including the testis, seminal vesicle, prostate and bladder. There were also no differences in serum testosterone, FSH or LH levels between Znf230 KO and wild-type mice (Table 1, Figure 3A, B). $\mathrm{H} \& \mathrm{E}$ staining demonstrated that the testicular tissue of Znf230 KO mice was intact and that each developmental stage of male germ cells was normal (Figure 3C-H). No sig- nificant difference was detected in the shape and number of sperm isolated from the epididymides of $\mathrm{Znf} 230 \mathrm{KO}$ and C57BL/6J wild-type mice (Figure 3I, J and Table 1).

\section{Changes in the mRNA expression profile of Znf230 KO mice}

Our previous report proposed that Znf230 was a DNA-binding protein that may function as a transcriptional activator (Qiu et al., 2003). We therefore investigated the differences between the transcriptomes of testes from Znf230 KO and wild-type mice using Affymetrix Mouse Genome 4302.0 Arrays. The expression levels of over 34,000 genes were assessed, and transcripts with fold changes greater than 2 or less than 0.5 between the two groups were analyzed (Table 2). Znf230 had the most down-regulated expression level of the analyzed transcripts, thereby confirming the validity of the experimental system. The mRNA level of one unclassified transcript 4921513I08Rik (GenBank No. AK014883) was detected to be more than two-fold higher, and the mRNA levels of three transcripts including two protein coding genes Kctd14 (GenBank No. NM_001136235) and 4930448A20Rik (GenBank No. Ak015411) and a noncoding RNA 4931431B13Rik (GenBank No. NR_045183) were detected to be more than two-fold lower in $\mathrm{Znf} 230$ $\mathrm{KO}$ mice compared with $\mathrm{C} 57 \mathrm{BL} / 6 \mathrm{~J}$ wild-type mice. Because the Kctd14 gene may encode a functional protein, we performed qRT-PCR analysis to verify the expression changes of Kctd14 between Znf230 KO and wild-type mice. The results showed that the mRNA level of Kctd14 
Table 1 - Comparison of phenotypes between Znf230 KO and C57BL/6J mice.

\begin{tabular}{|c|c|c|c|c|}
\hline & & Znf230 KO & $\mathrm{C} 57 \mathrm{BL} / 6 \mathrm{~J}$ & $\mathrm{P}^{\mathrm{a}}$ \\
\hline Total body weight $(\mathrm{g})^{\mathrm{b}}$ & & $30.18 \pm 1.45$ & $30.31 \pm 2.07$ & NS \\
\hline \multirow[t]{9}{*}{ Organ weight $(\mathrm{mg})^{\mathrm{b}, \mathrm{c}}$} & Brain & $434.55 \pm 30.71$ & $405.38 \pm 44.78$ & NS \\
\hline & Heart & $151.8 \pm 16.08$ & $139.54 \pm 8.48$ & NS \\
\hline & Lung & $171.21 \pm 12.93$ & $166.14 \pm 11.21$ & NS \\
\hline & Liver & $1382.53 \pm 82.92$ & $1386.72 \pm 192.7$ & NS \\
\hline & Kidney & $212.32 \pm 25.95$ & $204.1 \pm 21.18$ & NS \\
\hline & Spleen & $60.37 \pm 7.17$ & $63.64 \pm 8.29$ & NS \\
\hline & Seminal vesicle & $268.2 \pm 14.91$ & $236.1 \pm 40.69$ & NS \\
\hline & Prostate and Bladder & $127 \pm 25.22$ & $127.5 \pm 22.3$ & NS \\
\hline & Testis & $108.5 \pm 15.46$ & $109.6 \pm 23.67$ & NS \\
\hline Total no. of sperm $\left(\times 10^{6}\right)^{b}$ & & $24.4 \pm 3.6$ & $23.7 \pm 2.8$ & NS \\
\hline \multirow[t]{3}{*}{ Serum sex hormone $(\mathrm{ng} / \mathrm{mL})^{\mathrm{b}}$} & Testosterone & 1.290 .25 & $1.2 \pm 0.34$ & NS \\
\hline & Luteinizing hormone (LH) & $8.02 \pm 1.37$ & $8.21 \pm 1.72$ & NS \\
\hline & Follicle-stimulating hormone (FSH) & $10.2 \pm 3.05$ & $9.56 \pm 2.45$ & NS \\
\hline Litter size $^{\mathrm{d}}$ & & $7.5 \pm 1.4$ & $8.1 \pm 1.2$ & NS \\
\hline Life Span (days) $^{\mathrm{e}}$ & & $713.2 \pm 147.6$ & $722.5 \pm 168.5$ & NS \\
\hline
\end{tabular}

${ }^{a}$ Statistical analysis was carried out by Student's $t$ test, NS: Not significant.

${ }^{\mathrm{b}}$ Fifteen-week-old mice were examine, $\mathrm{n}=5$ per group, Values are means \pm S.D.

${ }^{c}$ Wet weights of paired organs were averaged for each mouse, and the single value was used to calculate mean \pm S.D among same genotype.

${ }^{\mathrm{d}}$ Data are mean values derived from six breeding pairs for each genotype.

${ }^{\mathrm{e}}$ Data are mean values of 10 mice ( 5 male plus 5 female) per group.

was reduced by more than 10 -fold in $\mathrm{Znf} 230 \mathrm{KO}$ mice compared with wild-type mice (Figure 4).

\section{Discussion}

The present work was undertaken in an effort to define the physiological role of the testis-enriched gene Znf230 in mammalian spermatogenesis. We generated a null mutation in the Znf230 gene by homologous recombination in mouse ESCs, which were used to produce homozygous Znf230 KO mice. Mice that were homozygous for the mutation lacked the intact mRNA and protein in germ cells, but did not exhibit any detectable abnormality in body growth or spermatogenesis. The absence of abnormality in Znf230-null testes was unexpected because of the testisenriched expression pattern of Znf230. However, several explanations may account for the lack of a clear phenotype in Znf230 KO mice. First, the function of Znf230 may be dispensable for male fertility. As an example, $\mathrm{H} 1 \mathrm{t}$ is an $\mathrm{H} 1$ histone variant that is unique to late spermatocytes and round spermatids, but H1t-null mice have no discernible phenotype (Fantz et al., 2001). Another example is SPAG5, which is an Odf1-interacting protein that is specifically expressed during meiosis. The disruption of SPAG5 does not affect spermatogenesis or fertility (Xue et al., 2002). Other examples include testicular orphan nuclear receptor 2 gene (Shyr et al., 2002), testicular haploid expressed gene (Mannan et al., 2003), transition protein 2, proacrosin and histone H1.1 genes (Nayernia et al., 2003), the tumor suppressor LRPIb gene (Marschang et al., 2004), the UBC4testis gene (Bedard et al., 2005) and the testis-enriched

Table 2 - Genes differentially expressed in the testes of Znf230 KO mice

\begin{tabular}{lcccc}
\hline Gene ID & Gene symbol & Gene name & Fold change $^{\mathrm{a}}$ & ProbeSet ID $^{\mathrm{b}}$ \\
\hline 70875 & 4921513108Rik & RIKEN cDNA 492151308 gene & 2.9705 & 1432299_at \\
67150 & Znf230 & ring finger protein 141 & $0.445 / 0.1841 / 0.158$ & $1449086 \_$at $/ 1433655 \_$at/ 1449087_at \\
233529 & Kctd14 & potassium channel tetramerisation domain containing 14 & $0.4276 / 0.4054$ & $1426632 \_$at $/ 1426633 \_$at \\
70971 & 4931431B13Rik & RIKEN cDNA 4931431B13 gene & 0.4054 & 1430416_at \\
73993 & 4930448A20Rik & RIKEN cDNA 4930448A20 gene & 0.2394 & 1454205_at \\
\hline
\end{tabular}

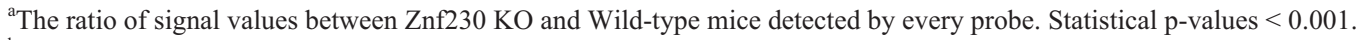

${ }^{\mathrm{b}}$ All probes hit one known transcript. 


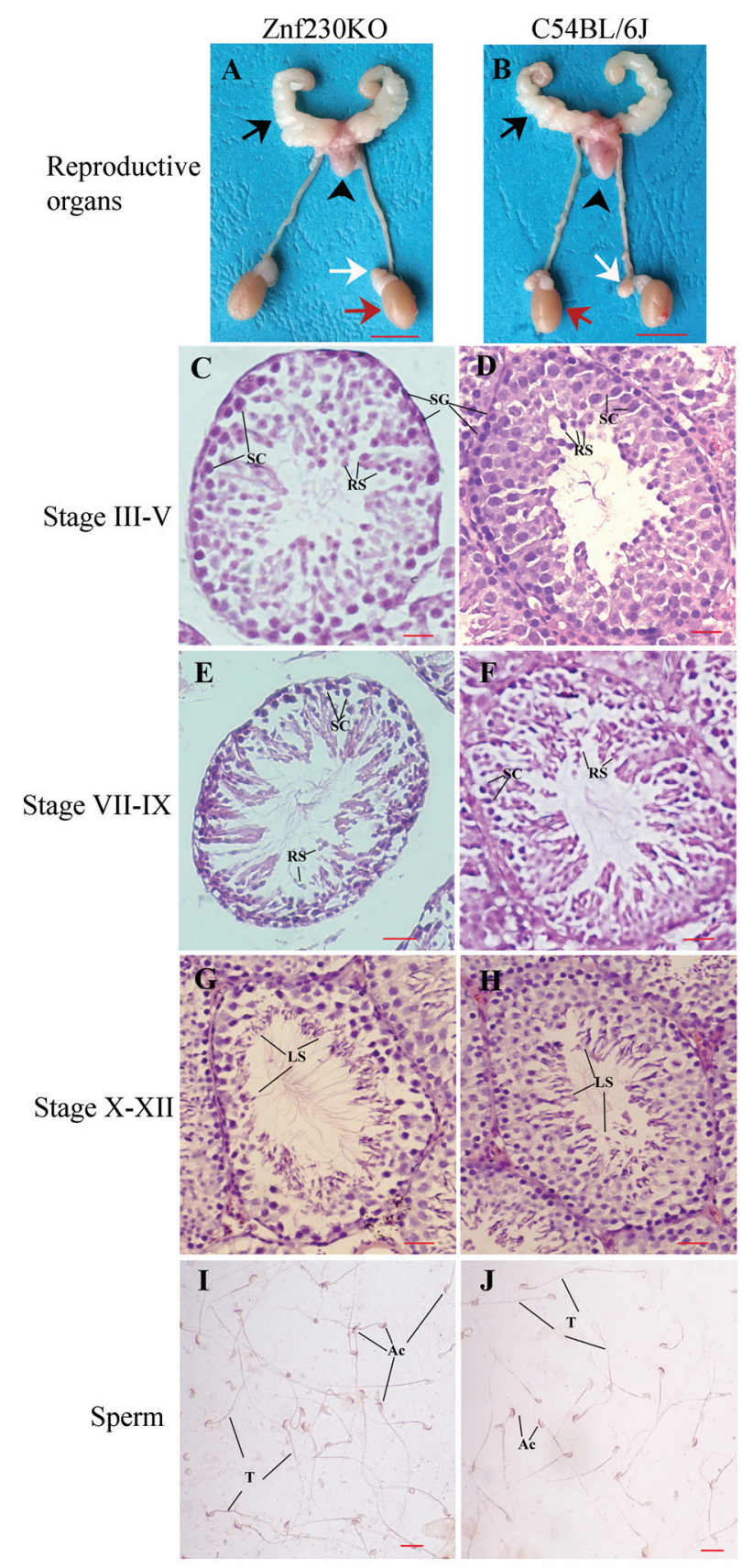

Figure 3 - Characteristics comparison of reproductive organs and sperm shape between Znf230 KO and C57BL/6J mice. Morphology of reproductive organs from Znf230 KO (A) and C57BL/6J (B) mice. The seminal vesicles (black arrows), bladder (black arrowheads), epididymis (white arrows) and testes (red arrows) were highlighted. Scale bar $=1 \mathrm{~cm}$. Histological analysis of $\mathrm{H} \& \mathrm{E}$ stained testes from Znf230 KO (C,E,G) and C57BL/6J (D,F,H) mice. SG: Spermatogonia, SC: Spermatocyte, RS: Round spermatids, and LS: elongated spermatids. Scale bar $=100 \mu \mathrm{m}$. Characteristics of H\&E stained sperm from Znf230 KO (I) and C57BL/6J (J) mice.. Ac: acrosome, T: sperm tail. Scale bar $=50 \mu \mathrm{m}$.

histone demethylase KDM4D gene (Iwamori et al., 2011). One explanation for such phenomena is that an unidentified protein may compensate for the loss of function of these genes. It is reasonable that other RING finger proteins may compensate for the loss of Znf 230 function because RING

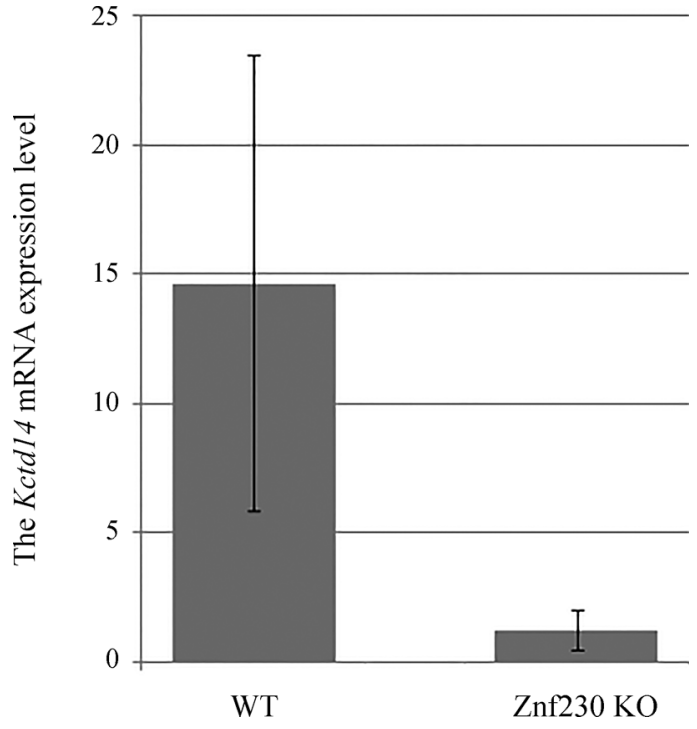

Figure 4 - Comparative qRT-PCR analysis of mRNA levels of the Kctd14 gene between Znf230 KO and C57BL/6J (WT) mice. Bars represent the means \pm S.D., Statistical p-values $<0.001$.

finger proteins belong to one of the largest zinc finger protein families. Second, the defects in Znf230 KO mice may be too small to be detected using the techniques employed here. A detailed ultrastructural examination of testicular tissues and spermatozoa may be needed to confirm eventual minor changes in the mutant mice. Third, because spermatogenesis is a very complex process that involves many genes, the inactivation of one gene may not be sufficient to produce a detectable phenotype. For example, Tyro 3, Axl, and $\mathrm{Mer}$ encode three structurally related receptors that possess tyrosine kinase activity. Mice that lack any single receptor or any combination of two receptors are viable and fertile, but males that lack all three receptors produce no mature sperm (Lu et al., 1999). Therefore, it will be of interest to explore the interaction between Znf230 and its related proteins by ablating their network and dissecting the resulting phenotypes to help clarify the biological role of the Znf230 gene in male fertility.

Indeed, we found that the mRNA levels of four transcripts were changed more than two-fold in Znf230 KO mice compared with $\mathrm{C} 57 \mathrm{BL} / 6 \mathrm{~J}$ wild-type mice. Three of the four transcripts were unclassified. However, Kctd14 encodes a putative member of the KCTD protein family that contains the bric-a-brac/tramtrak/broad (BTB) complex domain, which resembles the tetramerization domain of voltage-gated potassium channels. The KCTD protein family, which comprises 22 members, has been implicated in many important biological processes (Schwenk et al., 2010; Seddik et al., 2012; Cao-Ehlker et al., 2013; Skoblov et al., 2013). However, because no report has yet described the biological function of the Kctd14 protein, Znf230 KO mice may provide a clue for investigating the pathways in which these unclassified transcripts are involved. 
Conversely, the Znf 230 protein may act as a transcriptional factor, and the disruption of the $\mathrm{Znf} 230$ protein may cause the related transcriptional complex to be destroyed, this directly affecting the expression of the four transcripts. It is interesting to note that $Z n f 230$ and the four transcripts are all located on mouse chromosome 7 (Chr7). As shown in Figure S1, the transcripts of 4921513108Rik and Kctd14 are located upstream of Znf230, while the other two transcripts are located downstream of $Z n f 230$. It is thus possible that $c i s$-acting elements may have been destroyed during the targeted disruption of the Znf 230 gene. In addition, changes in the chromatin structure in the $\mathrm{KO}$ region of Chr7 may be the cause for the changes in the expression levels of the four transcripts.

In conclusion, we generated Znf230-deficient mice that exhibited normal body growth and fertility based on our current examination techniques. Using microarray analysis to compare the transcriptomes of testicular tissue from Znf230 KO and wild-type mice, we observed changes in the expression levels of four transcripts in Znf230 KO mice. In the future, the Znf230 KO mouse model may be used to uncover the biological roles and explore the interaction between $\mathrm{Znf} 230$ and its related transcripts.

\section{Acknowledgments}

This work was funded by a grant from the National Basic Program of China (Program 973) from the Ministry of Science and Technology of China (2012CB947600) and the Fundamental Research Funds for the Central Universities (2010SCU11025).

\section{References}

Bedard N, Hingamp P, Pang Z, Karaplis A, Morales C, Trasler J, Cyr D, Gagnon C and Wing SS (2005) Mice lacking the UBC4-testis gene have a delay in postnatal testis development but normal spermatogenesis and fertility. Mol Cell Biol 25:6346-6354.

Borden KLB (2000) RING domains: master builders of molecular scaffolds? J Mol Biol 295:1103-1112.

Chan W, Costantino N, Li R, Lee S, Su Q, Melvin D, Court DL and Liu P (2007) A recombineering based approach for high-throughput conditional knockout targeting vector construction. Nucleic Acids Res 35:e64.

Cao-Ehlker X, Zong X, Hammelmann V, Gruner C, Fenske S, Michalakis S, Wahl-Schott C and Biel M (2013) Upregulation of hyperpolarization-activated cyclic nucleotide-gated channel 3 ( $\mathrm{HCN} 3$ ) by specific interaction with $\mathrm{K}+$ channel tetramerization domain-containing protein 3 (KCTD3). J Biol Chem 288:7580-7589.

Fantz DA, Hatfield WR, Horvath G, Kistler MK and Kistler WS (2001) Mice with a targeted disruption of the H1t gene are fertile and undergo normal changes in structural chromosomal proteins during spermiogenesis. Biol Reprod 64:425-431.

Hwang K, Yatsenko AN, Jorgez CJ, Mukherjee S, Nalam RL, Matzuk MM and Lamb DJ (2011) Mendelian genetics of male infertility. Ann N Y Acad Sci 1214:E1-E17.
Irizarry RA, Hobbs B, Collin F, Beazer-Barclay YD, Antonellis KJ, Scherf U and Speed TP (2003) Exploration, normalization, and summaries of high density oligonucleotide array probe level data. Biostatistics 4:249-264.

Iwamori N, Zhao M, Meistrich ML and Matzuk MM (2011) The testis-enriched histone demethylase, KDM4D, regulates methylation of histone $\mathrm{H} 3$ lysine 9 during spermatogenesis in the mouse but is dispensable for fertility. Biol Reprod 84:1225-1234.

Jamsai D and O'Bryan MK (2011) Mouse models in male fertility research. Asian J Andro 13:139-151.

Joazeiro CAP and Weissman AM (2000) RING finger proteins: mediators of ubiquitin ligase activity. Cell 102:549-552.

Li SW, Arita M, Fertala A, Bao Y, Kopen GC, Långsjö TK, Hyttinen MM, Helminen HJ and Prockop DJ (2001) Transgenic mice with inactive alleles for procollagen $\mathrm{N}$ proteinase (ADAMTS-2) develop fragile skin and male sterility. Biochem J 355:271-278.

Liu P, Jenkins NA and Copeland NG (2003) A highly efficient recombineering-based method for generating conditional knockout mutations. Genome Res 13:476-484.

Liu Y, Tao D, Ma S, Kuang Y, Su D, Zhang H, Yang Y, Ma Y and Zhang S (2013) A new mutant transcript generated in Znf230 exon 2 knockout mice reveals a potential exon structure in the targeting vector sequence. Acta Biochim Biophys Sin 45:123-128.

Lu Q, Gore M, Zhang Q, Camenisch T, Boast S, Casagranda F, Lai C, Skinner MK, Klein R, Matsushima GK, et al. (1999) Tyro-3 family receptors are essential regulators of mammalian spermatogenesis. Nature 398:723-728.

Mannan AU, Nayernia K, Mueller C, Burfeind P, Adham IM and Engel W (2003) Male mice lacking the Theg (Testicular Haploid Expressed Gene) protein undergo normal spermatogenesis and are fertile. Biol Reprod 69:788-796.

Marschang P, Brich J, Weeber EJ, Sweatt JD, Shelton JM, Richardson JA, Hammer RE and Herz J (2004) Normal development and fertility of knockout mice lacking the tumor suppressor gene LRP1b suggest functional compensation by LRP1. Mol Cell Biol 24:3782-3793.

Nayernia K, Drabent B, Adham IM, Möschner M, Wolf S, Meinhardt A and Engel W (2003) Male mice lacking three germ cell expressed genes are fertile. Biol Reprod 69:1973-1978.

Qiu W, Zhang S, Xiao C, Xu W, Ma Y, Liu Y and Wu Q (2003) Molecular cloning and characterization of a mouse spermatogenesis-related ring finger gene znf230. Biochem Biophys Res Commun 306:347-353.

Russell LD, Ettlin RA, Sinha HAP and Clegg ED (1990) Mammalian spermatogenesis. In: Russell LD, Ettlin RA, Sinha HAP and Clegg ED (eds) Histological and Histopathological Evaluation of the Testis. Cache River Press, Saint Louis, pp 1-40.

Schlecht U, Demougin P, Koch R, Hermida L, Wiederkehr C, Descombes P, Pineau C, Jegou B and Primig M (2004) Expression profiling of mammalian male meiosis and gametogenesis identifies novel candidate genes for roles in the regulation of fertility. Mol Biol Cell 15:1031-1043.

Schultz N, Hamra FK and Garbers DL (2003) A multitude of genes expressed solely in meiotic or postmeiotic spermatogenic cells offers a myriad of contraceptive targets. Proc Natl Acad Sci USA 100:12201-12206. 
Schwenk J, Metz M, Zolles G, Turecek R, Fritzius T, Bildl W, Tarusawa E, Kulik A, Unger A, Ivankova K, et al. (2010) Native GABA(B) receptors are heteromultimers with a family of auxiliary subunits. Nature 465:231-235.

Seddik R, Jungblut SP, Silander OK, Rajalu M, Fritzius T, Besseyrias V, Jacquier V, Fakler B, Gassmann M and Bettler B (2012) Opposite effects of KCTD subunit domains on GABA(B) receptor-mediated desensitization. J Biol Chem 287:39869-39877.

Shyr CR, Collins LL, Mu XM, Platt KA and Chang C (2002) Spermatogenesis and testis development are normal in mice lacking testicular orphan nuclear receptor 2. Mol Cell Biol 22:4661-4666.

Skoblov M, Marakhonov A, Marakasova E, Guskova A, Chandhoke V, Birerdinc A and Baranova A (2013) Protein partners of KCTD proteins provide insights about their functional roles in cell differentiation and vertebrate development. Bioessays 35:586-596.

Song H, Su D, Lu P, Yang J, Zhang W, Yang Y, Liu Y and Zhang $\mathrm{S}$ (2008) Expression and localization of the spermatogenesis-related gene, Znf230, in mouse testis and spermatozoa during postnatal development. BMB Rep 41:664-669.

Xue J, Tarnasky HA, Rancourt DE and van Der Hoorn FA (2002) Targeted disruption of the testicular SPAG5/deepest protein does not affect spermatogenesis or fertility. Mol Cell Biol 22:1993-1997.

Yatsenko AN, Iwamori N, Iwamori T and Matzuk MM (2010)

The power of mouse genetics to study spermatogenesis. J Andro 31:34-44.

Zhang S, Qiu W, Wu H, Zhang G, Xiao C and Yang J (2001) The shorter zinc finger protein ZNF230 gene message is transcribed in fertile male testes and may be related to human spermatogenesis. Biochem J 359:721-727.

\section{Supplementary Material}

The following online material is available for this article:

Figure S1- The locations of the transcripts of Kctd14, Znf230, 4921513I08Rik, 4930448A20Rik and 4931431B13Rik.

This material is available as part of the online article from http://www.scielo.br/gmb.

Senior Editor: Emmanuel Dias Neto

License information: This is an open-access article distributed under the terms of the Creative Commons Attribution License, which permits unrestricted use, distribution, and reproduction in any medium, provided the original work is properly cited. 